\title{
Structure of Vortices in Two-component Bose-Einstein Condensates
}

\author{
D. M. Jezek ${ }^{1,2}$, P. Capuzzi ${ }^{1}$, and H. M. Cataldo ${ }^{1,2}$. \\ ${ }^{1}$ Departamento de Física, Facultad de Ciencias Exactas y Naturales, \\ Universidad de Buenos Aires, RA-1428 Buenos Aires, Argentina \\ ${ }^{2}$ Consejo Nacional de Investigaciones Científicas y Técnicas, Argentina
}

We develop a three-dimensional analysis of the phase separation of two-species Bose-Einstein condensates in the presence of vorticity within the Thomas-Fermi approximation. We find different segregation features according to whether the more repulsive component is in a vortex or in a vortex-free state. An application of this study is aimed at describing systems formed by two almost immiscible species of rubidium- 87 that are commonly used in Bose-Einstein condensation experiments. In particular, in this work we calculate the density profiles of condensates for the same conditions as the states prepared in the experiments performed at JILA [ Matthews, et al., Phys. Rev. Lett. 83, 2498 (1999)].

03.75. Fi, 67.57.Fg, 67.90.+z

Vortices in Bose-Einstein condensates (BECs) have thoroughly been studied from a theoretical point of view since the first experiments on condensates were carried out, due to their direct connection to superfluidity. For a review of these issues see for example [1], and for the most recent works references in [2]. But until the experiment of Matthews et al. [3] was performed, no evidence about their existence had ever been reported. This experiment was based on a method proposed by Williams and Holland [t] to create vortices in two-component BECs. They trapped atoms of ${ }^{87} \mathrm{Rb}$ in two different hyperfine states $\left|F=1, m_{f}=-1\right\rangle$ and $\left|F=2, m_{f}=1\right\rangle$, henceforth denoted as $|1\rangle$ and $|2\rangle$, respectively, and created vortices either in the $|1\rangle$ or $|2\rangle$ component, while the other species remained without rotation. Because of the relation between intra- and inter-particle scattering lengths, these states have the property of being almost immiscible. We shall see that this fact has important consequences on the structure of the vortices.

In connection with this experiment interesting theoretical work has recently been developed [5] in order to describe different properties of many types of twocomponent condensate systems. García-Ripoll and Pérez García, by means of a robust formalism, analyzed the stability and dynamics of a variety of two-species condensates, taking into account the effect of the interchange of species in the system, and also the variation of the number of particles. In particular, in Ref. [5] they set the relative number of particles equal in both species and varied the total number of particles, and in Ref. [6] they pursued the research by also varying the relative composition using a 2D model. However, in these works [5] 6] little is said about the structure of the stationary states and no comments are made about segregation of species. In a recent work, Chui et al. [7] computed the energy of different configurations within the Thomas-Fermi Approximation (TFA). They varied the number of particles and interchanged the species in order to derive conclusions about the relative stability. But, although they used a 3D model and considered phase separation in the mixture, a hypothesis was made regarding the form of the interface between species that we find is not consistent with our results and apparently also with new available experimental data [8]. We shall comment on this later.

In 1996, in a pioneering work, Ho and Shenoy [9] constructed an algorithm to determine the density profiles of binary mixtures of alkali atoms within the TFA. We develop here a different procedure which allows one to obtain conclusions about the general features of segregation through simple calculations.

The aim of the present article is to analyze and perform a classification of the structure of condensates near phase separation, when two almost immiscible species are involved. Our starting point for calculating the stationary states are the Gross-Pitaevskii equations in the TFA [1]:

$$
\begin{aligned}
& \mu_{1} \Psi_{1}=\left(V_{1}+N_{1} G_{1,1}\left|\Psi_{1}\right|^{2}+N_{2} G_{1,2}\left|\Psi_{2}\right|^{2}\right) \Psi_{1}, \\
& \mu_{2} \Psi_{2}=\left(V_{2}+N_{1} G_{1,2}\left|\Psi_{1}\right|^{2}+N_{2} G_{2,2}\left|\Psi_{2}\right|^{2}\right) \Psi_{2},
\end{aligned}
$$

where $N_{i}$ denotes the number of atoms of species $i$, and $G_{k, l}=u_{k, l} U$, with $u_{k, l}$ the relative interaction strengths between species $k$ and $l$. We set the most repulsive component $|1\rangle$ in the $\Psi_{1}$ state, and fix $u_{1,1}=1\left(>u_{2,2}\right)$, so $U=\frac{4 \pi \hbar^{2} a}{M}, a$ being the scattering length of the $|1\rangle$ species and $M$ the atom mass.

For simplicity, we consider the system in a spherically symmetric trap and make a change of variables according to $\sqrt{\frac{M}{2}} w_{0} \vec{r} \rightarrow \vec{r}$, with $w_{0}$ the trap angular frequency. Thus the effective potentials $V_{i}$ written in cylindrical variables read

$$
V_{i}=r^{2}+z^{2}+\frac{\kappa_{i}}{r^{2}} .
$$

The first two terms correspond to the trapping potential, while the third includes the centrifugal term [9], $\kappa_{i}=\frac{m_{i}^{2} \hbar^{2} w_{0}^{2}}{4}$, with $m_{i}$ the number of quanta of circulation associated with the wave function for species $i$.

Let us first analyze what happens when $D \equiv u_{1,1} u_{2,2}-$ $u_{1,2}^{2}=0$. Physically the system starts undergoing phase 
separation. Mathematically, for both $\left|\Psi_{i}\right|^{2}>0$, Eqs. (1) become linearly dependent. So to have a compatible set of equations the following condition must hold:

$$
\frac{\mu_{1}}{G_{1,1}}-\frac{V_{1}}{G_{1,1}}=\frac{\mu_{2}}{G_{1,2}}-\frac{V_{2}}{G_{1,2}} .
$$

If we define $\beta_{2}=u_{1,2} / u_{1,1}$, the above equation can be rewritten as

$$
\frac{\mu_{2}-\beta_{2} \mu_{1}}{1-\beta_{2}}-r^{2}-z^{2}+\frac{\beta_{2} \kappa_{1}-\kappa_{2}}{\left(1-\beta_{2}\right) r^{2}}=0 .
$$

This equation defines different families of surfaces that we shall call $\mathcal{S}_{0}^{i}$, where $i$ labels the family. As was mentioned above, this is the only region of space where both $\Psi_{i}$ may be non-zero, so these surfaces necessarily determine the interfaces between species. Such families are characterized by the values of

$$
A=\frac{\mu_{2}-\beta_{2} \mu_{1}}{1-\beta_{2}}, \quad B=\frac{\beta_{2} \kappa_{1}-\kappa_{2}}{\left(1-\beta_{2}\right)},
$$

(note that $D=0$ implies $\beta_{2}<1$ ).

Depending on the values of the above parameters, three topologically different kinds of surfaces exist, namely, spheres, cylinder-like surfaces, and toroids. In Fig. 1 we show a partition of the parameter space $(A, B)$ indicating the domains corresponding to each family. In order to classify these surfaces let us study the $z(r)$ curves defined by Eq. (4). If $z(r)$ intersects the $z$ axis, the associated surfaces $\mathcal{S}_{0}^{a}$ are spheres, and this occurs for $B=0$ and $A>0$. If $z(r)$ has only one root the corresponding surfaces $\mathcal{S}_{0}^{b}$ are cylinder-like, and this takes place when $B>0$. Finally, if $z(r)$ has two roots the related surfaces $\mathcal{S}_{0}^{c}$ are toroids, and it is easy to verify that the conditions to be fulfilled are $A^{2}+4 B>0, A>0$, and $B<0$. The remaining region of the parameter space has no associated surfaces.

To perform a displacement of a point within the parameter space two mechanisms are possible. On the one hand, the $A$ value may be changed by varying the number of particles in each species, which will cause a change in the chemical potentials. A variation of this type does not change the family, but the shape of the surface is modified. For example, for $B>0$ one can get a straighter "cylinder" by moving from positive to negative $A$ values, and, in terms of the number of particles, this corresponds to a decrease in the $N_{2} / N_{1}$ ratio. On the other hand, $B$ can only take discrete values and it is modified by changing the vorticity.

It is worthwhile mentioning that the only possible realization for $B=0$, (and hence for $\mathcal{S}_{0}^{a}$ ), within reasonable values of $m_{i}$, is a vorticity free condensate $m_{1}=m_{2}=0$. In such a case, regarding the location of each species, it is easy to conclude that the energy minimum is obtained when the less repulsive component lies inside the sphere of radius $\sqrt{A}$, while the other species forms a spherical shell around it. In the presence of vorticity $(B \neq 0)$, the location of each species can be predicted from energetic considerations. The state with greater vorticity should be located in the region of space that excludes $r=0$, because of the centrifugal term.

From this simple analysis we may state that close to phase separation the presence of vorticity dramatically changes the segregation structure.

Now, let us consider the general case $D \neq 0$. The solution of Eqs. (1) can be easily obtained and has the following different expressions depending on whether there exists any overlap between the wave functions of both species. a) In the region where only one wave function is non-vanishing $\left(\left|\Psi_{i}\right|^{2} \neq 0\right.$ and $\left|\Psi_{k}\right|^{2}=0$, for $\left.i \neq k\right)$ Eqs. (11) are decoupled and the solution is

$$
\left|\Psi_{i}\right|^{2}=\left[\mu_{i}-r^{2}-z^{2}-\kappa_{i} / r^{2}\right] /\left(G_{i, i} N_{i}\right) .
$$

b) In the region where both wave functions are nonvanishing, $\left|\Psi_{i}\right|^{2}>0$, one obtains

$$
\begin{aligned}
& \left|\Psi_{1}\right|^{2}=\left[\frac{\mu_{1}-\beta_{1} \mu_{2}}{\left(1-\beta_{1}\right)}-r^{2}-z^{2}+\frac{\kappa_{2} \beta_{1}-\kappa_{1}}{r^{2}\left(1-\beta_{1}\right)}\right] A_{1} u_{2,2}, \\
& \left|\Psi_{2}\right|^{2}=\left[\frac{\mu_{2}-\beta_{2} \mu_{1}}{\left(1-\beta_{2}\right)}-r^{2}-z^{2}+\frac{\kappa_{1} \beta_{2}-\kappa_{2}}{r^{2}\left(1-\beta_{2}\right)}\right] A_{2} u_{1,1},
\end{aligned}
$$

where $\beta_{1}=\frac{u_{1,2}}{u_{2,2}}$ and $A_{i}=\frac{\left(1-\beta_{i}\right)}{U N_{i} D}$.

We call $\mathcal{S}_{1}\left(\mathcal{S}_{2}\right)$ the surface defined by equating the expression inside the square bracket in $\left|\Psi_{1}\right|^{2}\left(\left|\Psi_{2}\right|^{2}\right)$ to zero. It is interesting to notice that the expressions mentioned above are of the same type as the one that defines $\mathcal{S}_{0}$ (cf. Eq. (4)), and so a similar classification can be applied to these surfaces. The surfaces $\mathcal{S}_{1}$ and $\mathcal{S}_{2}$ define the boundary of the coexisting region of the $|1\rangle$ and $|2\rangle$ species. Depending on the value of the interparticle interaction strength, the regions where the overlapping of the waves function occurs exhibit different features. For $D>0$ and $u_{1,2}$ small enough, the coexisting region can be very large, as may be observed in the figures shown in Ref. [9]. When increasing $u_{1,2}$ this region becomes narrower. At $D=0$, it is easy to verify that $\mathcal{S}_{1}=\mathcal{S}_{2}=$ $\mathcal{S}_{0}$, and hence the coexisting region is reduced to a single surface. When $u_{1,2}$ increases making $D<0$, this surface splits into two distinct surfaces, forming thus a new narrow $3 \mathrm{D}$ overlapping region, due to mutual repulsion of the species.

To have the problem completely solved one has to calculate the chemical potentials. In order to obtain $\mu_{1}$ and $\mu_{2}$ the normalization condition for the wave functions given by Eqs. (6) and (7) must be used. This leads to a system of two coupled integral equations that must be solved numerically. We have accomplished this in order to describe the states obtained in the JILA experiment [3], choosing parameters equal to those of the experiment. Namely, the trapping potential has an angular frequency $w_{0}=2 \pi \nu_{\text {trap }}$, being $\nu_{\text {trap }}=7.8 \mathrm{~Hz}$. The interaction strengths relative to the $|1\rangle$ component of the ${ }^{87} \mathrm{Rb}$ 
species are $u_{1,1}=1, u_{1,2}=.97$ and $u_{2,2}=.94$. And the number of particles of each species is $N_{1}=N_{2}=4 \times 10^{5}$. Note that for these values of the interaction strengths, $D=-9 \times 10^{-4}$ is close to the phase separation value, so the region between interfaces is very narrow, and hence $\mathcal{S}_{1} \sim \mathcal{S}_{2} \sim \mathcal{S}_{0}$. Thus hereafter, in order to identify the type of segregation of species encountered, we will make use of the classification we performed for $\mathcal{S}_{0}$.

For completeness, we also include calculations for the two species without vorticity, although the experimental realization consisted only of one vortex and one nonvortex state in each component.

In Fig. 2 we show the density contours of each wave function in the $(\mathrm{r}, \mathrm{z})$ plane for the following three configurations. (i) A vortex-free system $\left(m_{1}, m_{2}\right)=(0,0)$ yields chemical potentials in units of $\hbar w_{0}$ as $\mu_{1}=24.50$ and $\mu_{2}=24.06$. It may be seen that in this case the segregation evolves as $\sim \mathcal{S}_{0}^{a}$. The more repulsive component is on a spherical shell around the other, as expected. (ii) For $\left(m_{1}, m_{2}\right)=(1,0)$ we obtained $\mu_{1}=24.54$ and $\mu_{2}=24.04$. The vortex segregates in a ring surrounding the non vortex state. It is easy to verify that the species become separated by an interface of the type $\mathcal{S}_{0}^{b}$. The core size is about $9 \mu \mathrm{m}$ and the whole vortex has a radius of $26 \mu \mathrm{m}$. This reproduces very well the experimental data. (iii) When the vortex is in the less repulsive component $\left(m_{1}, m_{2}\right)=(0,1)$, we obtained $\mu_{1}=24.50$ and $\mu_{2}=24.15$. In this case the vortex is segregated inside the non-vortex state. The corresponding surface that describes such segregation is the toroid $\mathcal{S}_{0}^{c}$. The inner and outer radii of the torus are $5 \mu \mathrm{m}$ and $18 \mu \mathrm{m}$, respectively. However, this does not correspond to what was obtained at the first stage of the experiment, which looks quite similar to the pattern described in (ii). Mainly, in the experiment, the radius of the vortex was approximately $26 \mu \mathrm{m}$, while according to our calculations it should be $18 \mu \mathrm{m}$. Taking into account that after vortex creation, the vortex immediately shrank, a possible and likely explanation for the discrepancy is that the system was not created near the equilibrium configuration but moved towards it. The case with a vorticity of $\left(m_{1}, m_{2}\right)=(1,0)$ is created much closer to equilibrium 10. It is important to notice that in every case, even when the vortex configuration is out of equilibrium, the whole condensate is almost spherical with the same radius value of $26 \mu \mathrm{m}$.

In Fig. 3 we display the density profiles as a function of the radial coordinate at $z=0$, for the same three configurations described in Fig. 2. It may be seen in all of the cases that the shape of the total density is like a parabola, a characteristic that also holds for any other direction considered. Regarding the densities of each one of the components separately, they abruptly go to zero in the narrow region between species. It is worthwhile to mention that this behavior would not be so sharp for real systems and some interpenetrating region would exist 11.
With respect to the hypothesis made by Chui et al. [7], they assumed for condensates including vorticity in a symmetric trap that one component is confined inside a ball and the other one in a spherical shell around it; and that the species with vorticity have a small healing length sized vortex core. In the context of our article this means that they worked as if segregation of species, including vortices, always evolves as $\sim \mathcal{S}_{0}^{a}$, which is not consistent with our results. In a very recent experimental work \& Anderson et al. have included a figure (Fig. 3) in which a two-component vortex is viewed along its axis direction and along an orthogonal one. Views of a vorticity free condensate are also included. The in-trap pictures of Ref. [8] cannot rule out a healing length core because such a feature would be too small $(\sim 0.7 \mu \mathrm{m})$ to resolve. So in this experiment Chui's condensates should look like a vortex free condensate. As it may be seen in the above mentioned figure the images of a system with and without vorticity are quite different. In particular, we want to note that in the vortex case there is a visible evidence of a cylinder type segregation. However to be conclusive about these statements more experimental information is required.

In Refs. [5, 6] the authors employed, for describing the stationary states, wave functions expanded on a finite subset of the harmonic oscillator basis. This means that they did not work with exact solutions; unfortunately in these articles there is no precise information about the structure of the states. However, looking at both the domains of the wave function (Fig. 2) and the form of the density itself (Fig. 3), it seems that they should have taken into account a large number of elements of this basis to describe similar configurations.

It is well known that the TFA turns out to be very accurate when systems with a large number of particles are considered, so the main features of our analysis could be regarded as a guide to check the accuracy of different approximations.

Finally, we want to mention that very interesting experimental 12 and theoretical [13] work dealing with off-centered vortices in two-species condensates, is forthcoming.

In conclusion, we think that our analysis of states within the TFA could be helpful both from the experimental and theoretical viewpoints. In the first case, to prepare a configuration as close as possible to a stationary state, and in the second, to make a suitable choice for the set of wave functions in which such kinds of states are to be expanded.

We gratefully acknowledge useful conversations with B. P. Anderson. 
[1] F. Dalfovo, S. Giorgini, L. Pitaevskii, and S. Stringari Rev. Mod. Phys. 71, 463 (1999).

[2] B. Jackson, J. F. McCann, and C. S. Adams, Phys. Rev. Lett. 80, 3903 (1998), S. Stringari, Phys. Rev. Lett. 82, 4371 (1999), B. Caradoc-Davis, R. Ballaguand, and K. Burnett, Phys. Rev. Lett. 83, 895 (1999), D. Feder, C. Clark, B. Schneider, Phys. Rev. Lett. 82, 4956 (1999), and A. L. Fetter and A. A. Svidzinsky, condmat/0102003.

[3] M. R. Matthews, B. P. Anderson, P. C. Haljan, D. S. Hall, C. E. Wieman, and E. A. Cornell, Phys. Rev. Lett. 83, 2498 (1999).

[4] J. Williams and M. Holland, Nature (London) 401, 568 (1999).

[5] J. J. García-Ripoll and V. M. Pérez-García, Phys. Rev. Lett. 84, 4264 (2000).

[6] V. M. Pérez-García and J. J. García-Ripoll, Phys. Rev. A. 62, 033601 (2000).

[7] S. T. Chui, V. N. Ryzhov, and E. E. Tareyeva, Phys. Rev. A. 63, 023605 (2001).

[8] B. P. Anderson, C. A. Regal, D. L. Feder, L. A. Collins, C. W. Clark, and E. A. Cornell, Phys. Rev. Lett. 86, 2926 (2001).

[9] Tin-Lun Ho and V. B Shenoy, Phys. Rev. Lett. 77, 3276 (1996).

[10] B. P. Anderson, private communication.

[11] D. S. Hall, M. R. Matthews, C. E. Wieman, and E. A. Cornell, Phys. Rev. Lett. 81, 1543 (1998).

[12] B. P. Anderson, P. C. Haljan, C. E. Wieman, and E. A. Cornell, Phys. Rev. Lett. 85, 2857 (2000).

[13] S. A. McGee and M. J. Holland, cond-mat/0007143.

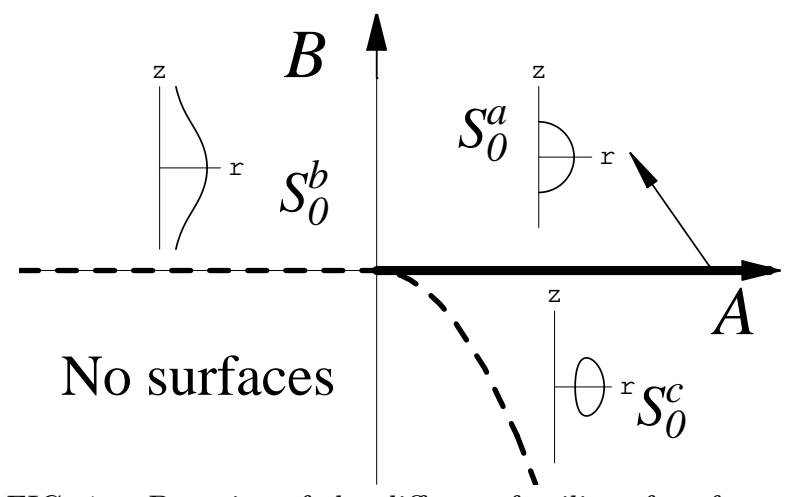

FIG. 1. Domains of the different families of surfaces $\mathcal{S}_{0}^{i}$ in parameter space $(A, B)$. In each region we indicate the related $z(r)$ curve. The thick line $B=0$ and $A>0$ corresponds to spheres. The $B>0$ region corresponds to cylinder-like surfaces. And for the conditions $A>0, B<0$, and for $B>-\frac{A^{2}}{4}$ the surfaces are toroids. The dashed line determines the separation between these families of surfaces and empty sets.
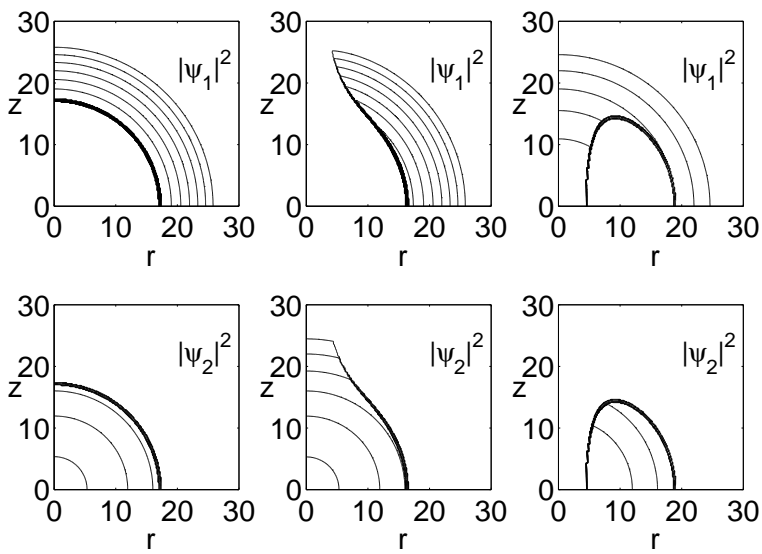

FIG. 2. Density contours in the $(\mathrm{r}, \mathrm{z})$ plane for component $|1\rangle(|2\rangle)$ in the first (second) row of subfigures. The columns correspond to the following situations: (left) no vortex $\left(m_{1}, m_{2}\right)=(0,0)$, (middle) a vortex in $|1\rangle$ species $\left(m_{1}, m_{2}\right)=(1,0)$, and (right) a vortex in the less repulsive component $\left(m_{1}, m_{2}\right)=(0,1)$. The density contour spacing is $10^{-5} \mu \mathrm{m}^{-3}$.
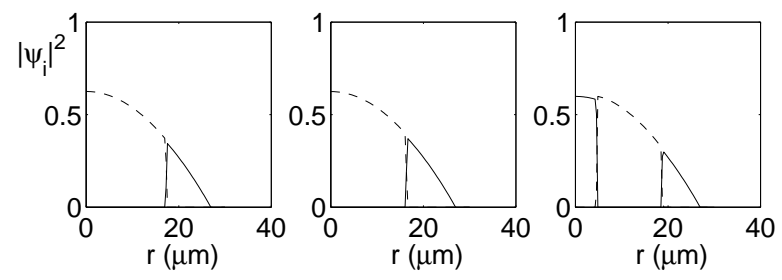

FIG. 3. Density profiles $\left|\Psi_{1}\right|^{2}$ (solid line) and $\left|\Psi_{2}\right|^{2}$ (dashed line) as function of $r$ at $z=0$ for the same three distributions of vorticity described in the previous figure. The density is given in units of $10^{-4} \mu \mathrm{m}^{-3}$. 